\title{
Architecture and Protocols for Toll-Free Electric Vehicle Charging
}

\author{
Raziq Yaqub \\ Department of Electrical Engineering and Computer Science, Alabama A\&M University, Normal, AL 35763, \\ USA; raziq.yaqub@aamu.edu
}

Received: 25 August 2018; Accepted: 20 February 2019; Published: 24 February 2019

\begin{abstract}
This paper proposes system architecture and protocols for the deployment of a toll-free electric vehicle charging service. The architecture enables the party initiating the electric vehicle (EV) charging to have their service request authorized by the system and paid for by a third party.
\end{abstract}

Keywords: toll-free EV charging; authentication; authorization; accounting; policy; charging rules

\section{Introduction}

Electric vehicle (EV) charging stations are being deployed worldwide [1] commercially at street parking, gas stations, service areas on highways, taxi stands, in the parking lots of hotels, airports, shopping centers, convenience shops, restaurants, coffee houses, parks, train stations, office buildings, etc.

Just as when they patronize other businesses, EV drivers may benefit from two payment models to charge their EVs. In one model, the user pays from their own account (cash, check, service subscription, credit cards, etc.), and in the other model, the user may have a business or a community pay for the service.

Since EV charging (3-6 cents/mile) is cheaper than gasoline (12-16 cents/mile), businesses or communities may benefit from leveraging it as a marketing tool, and they may wish to a provide toll-free charging service to their patrons.

The following scenarios are foreseen:

1. Advertisers and community organizers will attract clients and communities to business and community locations by offering toll-free charging incentives.

2. Retailers/wholesalers will include toll-free charging in the service portfolio to promote sales or services.

3. Car rental lots/dealers will offer or package a value-added service by providing toll-free charging.

4. Organizations and communities, e.g., the employers, the profit/non-profit organizations, and the fleet management companies, will provide toll-free charging to the field force/communities to streamline their accounting procedures. Communities may offer free charging to attract EV owners to the area of the community, which increases traffic and sales tax revenues.

5. A sign-up service portal, provided by the utility company, is also suggested. This would allow businesses and communities to track their service usage, the cost of consumed service, and their billing history over the internet. The service may be enabled on mobile devices as well, as Wi-Fi and $4 \mathrm{G}$ networks have become widely available. The portal not only provides the real time information about service consumption, cost, and billing history, but also the convenience of paying bills around-the-clock and the ability to monitor accounts more closely for early detection of fraudulent activity that might cause financial damage or loss to businesses or communities. 
The various companies, exemplified above, are referred to as "businesses" and "communities". The businesses and communities may have wholesale deals with utility companies to provide toll-free charging services to their clients and community members.

In the realm of telephone communication, toll-free telephony [2] has been globally successful in providing a party pay model that obviates the need for the calling party to pay for the service used. However, the concept of toll-free charging of EV (where a business or a community will pay for EV charging) has not yet been developed. There is an opportunity in the market for this model of EV charging payment. The purpose of this paper is to provide a system, method, and architecture that enables the party initiating the EV charging to have their service request authorized and paid for by the business or a community. In addition, the method will support conventional payment methods, in case the EV driver elects to pay for the service.

In this paper, the EV charging station capable of providing such services is called the TEC (Toll-Free EV Charging) station, the service is called toll-free service, and the number used to claim the service is called a toll-free number.

\section{Detailed Description}

Figure 1 presents a logical system architecture for supporting a toll-free service provisioning system. It involves the following logical and functional entities:

- Logical parts of a toll-free number

- $\quad$ TEC station

- $\quad$ TEC-Gateway/Router (TEC-GW)

- $\quad$ TEC-AAA (Authentication, Authorization, and Accounting) Server

- Policy and Charging Rules Server (PCRS)

- $\quad$ Subscription Profile Database (SPD) Type-1 (to support toll-free services)

- $\quad$ Subscription Profile Database (SPD) Type-2 (to support non-toll-free services)

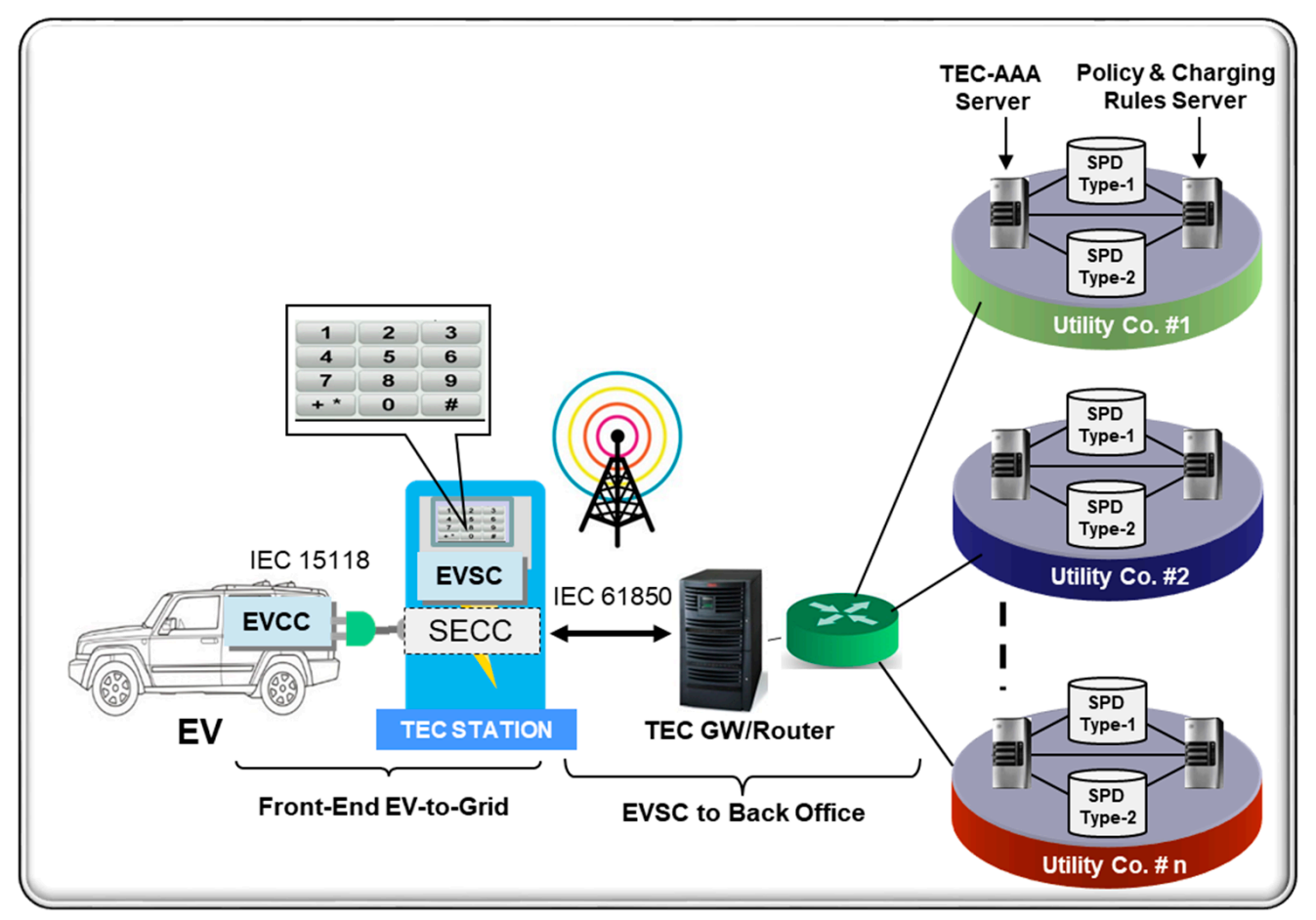

Figure 1. Architecture of toll-free electric vehicle charging (TEC) system. 


\subsection{Logical Parts of a Toll-Free Number}

A toll-free number is an IP address [3], a sequence of digits, or a code. It is specifically tailored to activate a toll-free service from the TEC station. A toll-free number may consist of several groups. In the context discussed in this paper, the toll-free number is structured so that the first part is analyzed and automatically translated by the TEC-GW/Router to uniquely identify the TEC-AAA server to establish a communication path between the TEC station and the TEC-AAA server. The TEC-AAA server resides in the utility company's network (examples of such networks include the Alabama electric company, Duke energy, Southern California Edison, etc.). Due to TEC data collection and communication abilities, it may become a target for malicious attackers who may benefit from stealing or manipulating the TEC data. To make the system attack proof, security mechanisms should be in place. These mechanisms include secure installation of TEC and secure communications between TEC, TEC GW/Router, and TEC AAA Server. The communications media may be wired and wireless, though some of the wireless networks may be more vulnerable.

Reference [4] provides a complete set of security requirements. The proposed system is architected to meet these requirements to provide strong security and privacy, through (a) the deployment of AAA and the policy and charging rules server, proposed in this paper, and (b) the end to end, between EV and back office, secure protocols, ISO15118, IEC 61850, and IEC63110, defined by the ISO/IEC and used for communication among these entities. Thus, the proposed system offers a robustly secure system. The AAA assures that a communicating entity is the one that it claims to be, and it also provides a fair billing for the home as well as the roaming EVs. It also offers a secure payment solution. The TEC GW/Router and AAA ensure that only the EVs authorized by a certain utility company are connected. The policy charging rules entity ensures that the EV is accounted only for the electricity used by the EV, according to the prevailing policies of the utility companies on a real-time basis. ISO/IEC protocols assure the authenticity of the messages. Reference [5] provides a complete security analysis of a smart electric vehicle charging.

The second part of the toll-free number is analyzed and automatically translated by the TEC-AAA server to uniquely identify the service associated with the number and the specific party to be billed. The second part, if associated with toll-free service, is depletable. This means that once the service associated with the number is consumed; the number is depleted or disabled and is no longer usable. It signifies the free of cost charging duration and the quantity of the free charging.

It is important to note that it may be free for the end user, but someone (advertisers, retailers, marketers, employers, etc.) will still be paying to the utility for service.

1. Advertisers/marketers will purchase or subscribe with the utility company for toll-free service to attract prospects to a target location (e.g., to a specific local business or a community) by providing toll-free charging.

2. The retailers will purchase or subscribe with the utility company for toll-free service to promote their sales and/or services by providing toll-free charging to their clients.

3. Car dealerships/rental lots will purchase or subscribe with the utility company for toll-free service to offer value-added service by providing toll-free charging to the drivers/travelers.

4. Employers, nonprofit organizations, and fleet management companies (police, trucking, public service vehicles, etc.), will purchase or subscribe with the utility company for toll-free service for their field force. The field force may belong to the government, nongovernment, or private organizations/communities. It may include (a) a team capable of providing a tactical force for a wide variety of policing or emergency response functions, (b) a staff of customer-focused individuals supporting administration, inspecting, monitoring, scheduling, and shifting of goods, (c) a group of community social workers who perform a broad spectrum of duties ranging from providing support/treatment to individuals with diseases, emotional distress, behavioral or mental health disorders. 
The first part of the toll-free number, if not associated with toll-free service, serves as an identifier and is not depletable. It is the utility consumer's/EV driver's account number and signifies the consumer to be billed against that number, e.g., it may be a consumer's home, business, or a community utility bill account number. The format of the toll-free number (be it an IP address, a sequence of digits, or a code), may be standardized in ways that the entities involved in toll-free service may distinguish for each group of toll-free numbering schemes.

The addressed utility company's TEC-AAA server validates the toll-free number and associated service type by consulting databases, policy servers, billing management systems, etc. Upon validation, the TEC station receives a signal to activate the electrical circuit connection and start charging the EV battery. The signal is received through the TEC-GW/Router. During the charging process, the TEC station and the TEC-AAA server exchange messages through the TEC-GW/Router to ensure that the EV gets the service it deserves.

\subsection{TEC Station}

TEC stations are smart, toll-free enabled EV chargers. They are distributed throughout the service area to provide a charging service. In addition, they are capable of communicating with a host electrical utility and other participating electrical utilities. Each TEC station has a unique ID, which may be either a fixed IP address or a temporary one, such as an address allocated to the TEC station for a single session. Allocation of a temporary address can be supported by the Dynamic Host Configuration Protocol (DHCP) defined by the Internet Engineering Task Force (IETF) [6]. The TEC station includes all standard features, and some additional features explained in the following paragraphs:

The TEC station is capable of communicating its ID and charging capabilities to the TEC-GW/Router. The communication may be carried out using any state-of-the-art protocols, such as Internet Protocols (IP), and through any state-of-the-art transmission technology such as 4G/5G, or optical fiber.

The TEC station is capable of receiving and parsing a toll-free number, which is essentially an IP address/message. Thus, TEC stations would enable the user to dial a toll-free number. The dialing mechanism may be enabled by (a) integrating a hardware/software dialing pad built into the TEC station, or (b) integrating a scanner that could scan the IP address, or read the IP address using RFID/NFC (Radio Frequency ID/Near Field Communication) technologies. This would eliminate the need for entering the numbers manually.

A toll-free number may be human readable digits, or it may be a machine-readable barcode, or a $\mathrm{QR}$ code, etc. It may be delivered by a business or a community to an EV driver in several possible ways, outlined below:

- A printed toll-free number on a card issued by a business or a community delivered by hand

- A printed toll-free number on a shopping receipt delivered at the point of sale after the EV driver had shopped any product or service

- An electronic toll-free number delivered via e-mail or text message

- An electronic toll-free number read by the mobile device through its scanning capabilities via NFC/RFID, thus eliminating the need to actually dial.

The TEC station, after receiving the toll-free number provided (in whatever format) by the EV driver, will then forward it to the TEC-Gateway and router.

\subsection{TEC-Gateway/Router}

The TEC-Gateway/Router is part of the utility service provider's network. Each toll-free-GW/Router has state-of-the-art features that any communication GW and Router may have. It has the capability of coordinating and managing all the toll-free service-related tasks and is capable of handling messages and tasks in a standard way.

The TEC-GW/Router forwards the toll-free number and message in the form of data packets across several functional entities. These entities may reside in the host electrical utility company's 
network, other participating electrical utility companies' networks, and/or in the application service providers' network.

When data comes in from the TEC station side, the TEC-GW/Router reads the address information in the IP packet to the network side. In other words, it determines and forwards all IP packets to the destined ultimate TEC-AAA server or application server. Similarly, when data comes in from the network side, such as from the TEC-AAA server, or application server, it reads the address information in the IP packet to determine its destined TEC station.

There are two types of data transferred by a TEC; i.e., the power consumption data, and the business/community subscription data. These two sets of data are collected on each charging event and stored in utility databases. The data may be compromised by the internal players (such as TEC owners, or power company workers), or the external actors (such as cyber hackers, or service users who may perform malicious actions with TEC at the site). The most significant attacks that threaten information security and privacy are injecting malicious software in TEC, altering data on its flight, and illegal access to databases.

To mitigate attacks on data during its flight, the system uses secure communication protocols designed by IETF (Internet Engineering Task Force) such as IPSec (Internet Protocol Security), SSL (Secure Socket Layer), TLS (Transport Layer Security), and SSH (Secure Shell), state-of-the-art encryption, firewalls, and user access control mechanisms. These tools provide data confidentiality, integrity, and authenticity between all nodes in network communication. The gateway security is always a concern and, therefore, a reliable gateway time is necessary. To achieve a reliable gateway time and to avoid, detect, or mitigate attacks, the TEC gateway supports Intrusion Detection and Prevention Systems (IDS/IPS), signature detection, anomaly detection, and packet filtering (Firewall) which can monitor and filter the network traffic.

\subsection{TEC-AAA (Authentication, Authorization, and Accounting) Server}

The TEC-AAA Server's authentication function verifies the toll-free number to determine if the toll-free number is a legitimate number. The TEC-AAA Server's authorization function determines whether a toll-free number is authorized to use a certain service. This authorization may be determined based on a range of restrictions, such as time-of-day restrictions, physical location restrictions, or restrictions against multiple accesses by the same entity. This authorization can be made contingent on additional conditions such as event-based charging, online charging, offline charging, toll-free charging, non-toll-free charging, etc. Accounting refers to the tracking of network resource consumption by users primarily for the purpose of billing.

To perform the above-noted authentication, authorization, and accounting functions, the TEC-AAA server interacts with the PCRS, SPD Type-1, and SPD Type-2, in addition to the TEC-GW/Router.

The TEC-AAA server may bill the business or a community on real-time, or on batch accounting. This means that it may be coded to deliver the accounting information concurrently with toll-free service usage and consumption of the resources, or in a batch, saving the accounting information until it is delivered at a later time. This can be modified to suit the unique accounting needs of the business or the community.

The TEC-AAA server may also perform some additional functions, such as capacity planning, trend analysis, and cost allocation. Information that is gathered in accounting is the identity of the user or subscribing business or community, the nature of the service delivered, the volume of usage, time duration, including when the service began and when it ended, etc. The TEC-AAA server may also record events such as authentication and authorization failures. It may also perform auditing functions, verifying the correctness of procedures carried out based on accounting data. TEC documents the consumption, history, and the trends, and reports it to the utility company. Attacks on personal data may be triggered by unauthorized access. These attacks can be mitigated, and privacy can be preserved by limiting the access to the select authorized users, implementing novel encryption techniques, applying intrusion detection systems, using trusted platform modules in TEC and trusted 
third parties (i.e., TEC sends encrypted data to the TTP, and TTP sends the anonymized or filtered data to the utility company). There are several other state-of-the-art techniques to ensure privacy, which can be seen in [7].

\subsection{Policy Charging and Rules Server (PCRS)}

The PCRS is located in each participating utility's network. However, for a given toll-free service, the policy of that PCRS applies to the utility network that provides the toll-free service. The PCRS determines and applies the policy and charging rules in real time. Therefore, the PCRS interfaces with the application function, specialized functions, real-time rate and the billing management server [1] to manage the service offering rate and billing on a real-time basis.

As detailed in [1], the rate adjustment is based on several factors such as special promotions, event-based services, customer loyalty membership, local market dynamics, geographical locations in the town, real-time electricity pricing based on system demand dynamics, generation versus load conditions, and peak/off-peak hours.

The PCRS aggregates information to and from the utility network, operational support systems, and other sources in real time, supporting the creation of rules and then automatically making intelligent policy decisions for each subscriber active on the network. This will be useful for the utility to efficiently control the distribution, maintain balance in the power system, and shave the peak demand [1].

Though this paper explains toll-free service, the architecture proposed may benefit a multitude of services. Application function can support applications such as toll-free service provisioning based on real-time participation in surveys, watching advertisements or promotional videos and responding to questionnaires, etc., as explained in Figure 4.

The PCRS owner can change and update policies dynamically. The PCRS owner can also dynamically instruct the PCRS on how to handle packets destined to the TEC station, or to other functional entities and databases. The PCRS owner can also dynamically instruct the PCRS on how to handle or reject the communication of an authorized and unauthenticated user.

\subsection{Subscription Profile Database (SPD) Type-1 and Type-2}

The PCRS also interfaces with Subscription Profile Databases Type-1 and Subscription Profile Databases Type-2.

The Subscription Profile Database Type-1 contains the database of businesses and communities who subscribed with the utility service provider to offer toll-free service to their clients. Type-1 subscribers might be employers, nonprofit management entities, marketers/advertisers, etc. Marketers may use it as a tool to lure in consumers to shop and entice them to their products and/or services by offering free charging service to their patrons. The PCRS may have different sets of policy and charging rules for such subscribers.

The Subscription Profile Database Type-2 contains the database of those non-business/community consumers, i.e., those consumers who do not subscribe for a toll-free service, but directly consume electricity themselves. These consumers might like to be billed to their existing account even for outdoor electricity usage, i.e., when they consume electricity while they are roaming in their town or other utility service provider's networks but prefer to have one electric bill.

The interface between the SPD Type- 1 and the PCRS allows the PCRS to request the subscriber's subscription information and the policies based on any specific toll-free number. The interface between the SPD Type-2 and the PCRS allows the PCRS to request the consumer's account information and policies tied to those account numbers. The SPD Type- 1 and SPD Type- 2 also notify any change in subscription profile to the PCRS.

\subsection{Usage Scenarios}

To understand the concept, let us consider the following four possible usage scenarios: 


\subsubsection{Usage Scenario 1}

Assume an EV driver carries a toll-free number issued by a business or a community that has a subscription with Southern California Edison (SCE). Also, assume that this EV driver, while visiting Tennessee, plugs his EV in the TEC station provided by Tennessee Valley Authority (TVA), and feeds the toll-free number. The TEC station directs the toll-free number to the TEC-GW/Router present in the host utility, TVA in this case. The TEC-GW/Router parses the first part of the toll-free number and finds that the number is issued by the SCE. Thus, the TEC-GW/Router routes the toll-free number to the TEC-AAA server residing in the SCE network in California. It may arise security and privacy concerns again, however, by having a roaming contract in place and security mechanisms implemented, as the cell phone service providers do, the security and privacy concerns can be resolved.

The TEC-AAA server of SCE analyzes the second part of the toll-free number and verifies (i) the legitimacy of the number and (ii) the service associated with it. To retrieve the associated services, it consults the SPD Type-1, which contains the database of businesses/communities and services. Once the toll-free number is determined to be a valid number, the TEC-AAA server of SCE sends "Authorization" and "Service description" (e.g., "s" kWh of Service or "\$" amount) for which the toll-free number is eligible to TVA's TEC-GW/Router. The TVA TEC-GW/Router then contacts TVA's PCRS. There, it receives updated information from several other stations, and may have a different set of policy and charging rules for each customer, such as one set of policies for SCE, another set of policies for Alabama Power, and another set of policies for its own local consumers. Using that information, it will find out the real-time service volume (in terms of " $\$$ " or $\mathrm{kWh}$ ) according to the prevailing policies of TVA. Upon computation of the real service volume (e.g, "s" kWh of Service), the TEC-GW/Router sends a command message to the TEC station in Tennessee to provide " $\mathrm{s}$ " $\mathrm{kWh}$ of the toll-free service to the visiting EV driver. After providing the service, the TEC-GW will send an invoice to the TEC-AAA server of SCE in California. SCE in California may deliver the amount to TVA either in real time or at the end of a certain period (batch invoices) according to the agreements.

This service is analogous to prepaid telephone service models, and all the features of that model of prepaid service (even those which are not specifically mentioned in this paper) can also be realized.

\subsubsection{Usage Scenario 2}

The reverse scenario is also possible.

Assume an EV driver holds a toll-free number issued or distributed by a company that is a subscriber of TVA, in Tennessee. Also, assume that this EV driver is visiting California. They plug their car into the TEC station provided by SCE and dial the toll-free number. The TEC station directs the toll-free number to the TEC-GW/Router present in the host utility (SCE in California, in this case). The TEC-GW/Router analyzes the first part of the toll-free number and finds that it belongs to TVA. The TEC-GW/Router routes the toll-free number to the TEC-AAA server residing in the TVA network in Tennessee. The TEC-AAA server of TVA analyzes the second part of the toll-free number and verifies (i) the legitimacy of the number and (ii) the service associated with it. To retrieve the associated services, it consults SPD Type-1 (SPD Type-1 contains the database of businesses and communities). Once the toll-free number is determined to be a valid number, it sends "Authorization" and "Service Volume" (e.g., "s" kWh of Service or "\$" amount) for which the toll-free number is eligible to the SCE TEC-GW/Router. SCE's TEC-GW/Router contacts SCE's PCRS to find out the real-time service volume in terms of " $\$$ " or $\mathrm{kWh}$, considering loading/peak condition according to the prevailing policies of TVA. The PCRS receives updated information from several other stations, and also may consult different sets of policy and charging rules for each customer such as one set of policies for TVA, another set of policies for Alabama Power, and yet another set of policies for its own local consumers. Upon computation of real service volume (say "s" kWh of Service), the TEC-GW/Router sends a command message to the TEC station in California to provide " $\mathrm{s}$ " $\mathrm{kWh}$ of the toll-free service to the visiting EV driver. After provision of service, the TEC-GW would send an invoice to the TEC-AAA 
server of TVA in Tennessee. TVA may deliver the amount to SCE either in real time, or at the end of a certain period (batch invoices), or according to the agreements.

As before, this service is analogous to prepaid telephone service models, and all the features of that model of prepaid service, (even those which are not specifically mentioned in this paper) can also be realized.

\subsubsection{Usage Scenario 3}

Now, assume an EV driver holds a toll-free number which is actually his Account Number issued by SCE. Also, assume that this EV driver is visiting Tennessee. He plugs his car into the TEC station provided by TVA, and dials the toll-free number. The TEC station directs the toll-free number to the TEC-GW/Router present in the host utility, i.e., TVA in Tennessee in this case. The TEC-GW/Router analyzes the first part of the toll-free number and finds that it belongs to SCE. The TEC-GW/Router routes the toll-free number to the TEC-AAA server residing in the SCE network in California. The TEC-AAA server of SCE analyzes the second part of toll-free number and checks (i) if the toll-free number is legitimate and (ii) the service credentials associated with it. For the associated service credential check, it consults the SPD Type-2, which contains the database of those non-business/community consumers who are not buying electricity for a toll-free Service, but who directly consume electricity themselves.

The rest of this process will proceed in the same manner as in usage Scenario 2.7.1. As before, this service is analogous to prepaid telephone service models, and all the features of that model of prepaid service, (even those which are not specifically mentioned in this paper) can also be realized.

\subsubsection{Usage Scenario 4}

Now, assume there is an EV driver who holds a toll-free number issued or distributed by a company that is a subscriber of the TVA. Also, assume that this EV driver is a graduate student of University of Alabama Agriculture and Mechanical University (AAMU). Their EV is estimated to be charged $60 \%$ (using toll-free service) in $40 \mathrm{~min}$. They are offered an option to participate in an AAMU Survey or watch a promotional advertisement of an Atlanta-based marketing company followed by questions to answer, to get a few extra minutes of EV charging. They think that while waiting, doing nothing, it might be a good use of time, so they agree to participate in an AAMU survey. The TEC-GW/Router asks the application function to present an AAMU general public level survey that could be completed in $40 \mathrm{~min}$. The application functions or application server (which may reside in AAMU or TVA's network) finds and presents the pre-saved promotional survey/questionnaire of AAMU that is estimated to take 30 to $40 \mathrm{~min}$. Based on the user's correct or attempted responses, an equivalent extent (e.g., 30\% extra) of toll-free charging service is added. The EV driver gets an aggregate of $90 \%$ of toll-free charging. TVA charges AAMU for $30 \%$ of charging, according to the agreements between AAMU and TVA.

These kinds of services, billing arrangements, ads provisioning, etc., would require an MoU (Memorandum of Understanding) in place between the companies and entities to support seamless roaming as well as advertisement-based service offerings to EV drivers across different utility providers.

\subsection{Protocol Implementations}

To support toll-free methodology as presented in this paper, signaling protocols to enable communication between different entities are needed (e.g., between the TEC station and TEC-GW/Router, the TEC-GW/Router and TEC-AAA server, and the TEC-AAA server and PCRS/SPDs). We propose to use existing well-established protocols. These protocols may be internet protocols (IP) and AAA [8] based on diameter [9], explained in IETF RFCs.

The architecture of Figure 1 shows the basic functional entities. As the service evolves, some other entities may also be introduced, such as real-time rate and billing management server, application 
function, application servers, operational support systems, etc., to give better services and better user experience.

\section{Flow Chart}

Figure 2 shows an algorithm for the resolution of the routing path and service type. The TEC station user interface prompts the EV Driver to input a toll-free number. The TEC station accepts the user input and sends the toll-free number to the TEC-GW/Router. The TEC-GW/Router analyzes the first part to uniquely identify the specific utility company and establishes a communication path between the TEC station and the TEC-AAA server of that utility company.

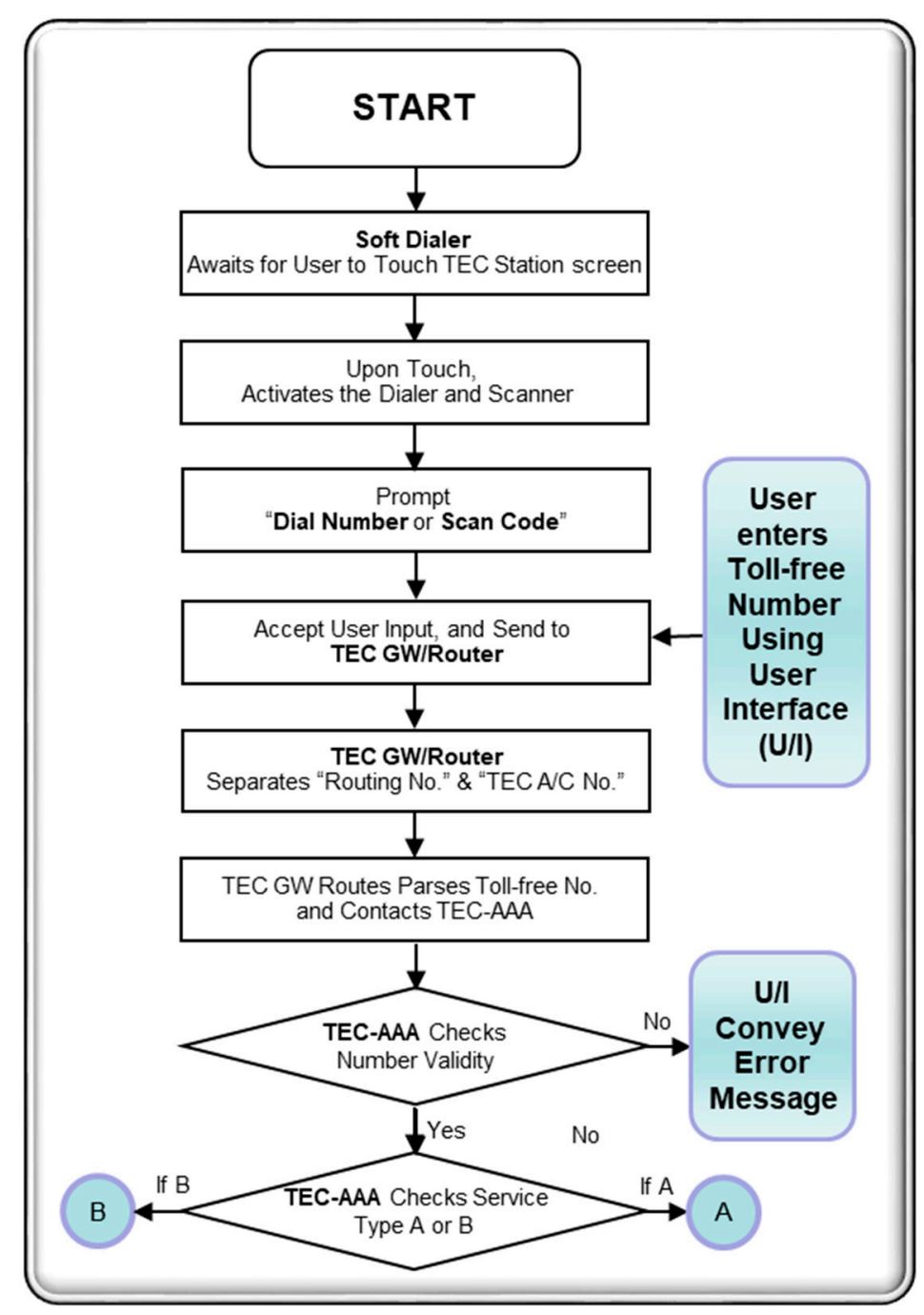

Figure 2. Routing, and resolution of service type.

The second part of the toll-free number is analyzed by the TEC-AAA server to authenticate the toll-free-number and identify the service type: Type-1 Service (wherein the cost is paid by a certain business or a community; toll-free for the end user) or Type-2 Service (wherein the charging cost is paid by the user from their own account). Each toll-free number may be associated with a unique utility service provider and unique service offering. This is explained in Figures 3 and 4.

Figure 3 shows a toll-free service provisioning that is verified by SPD-Type-1. 


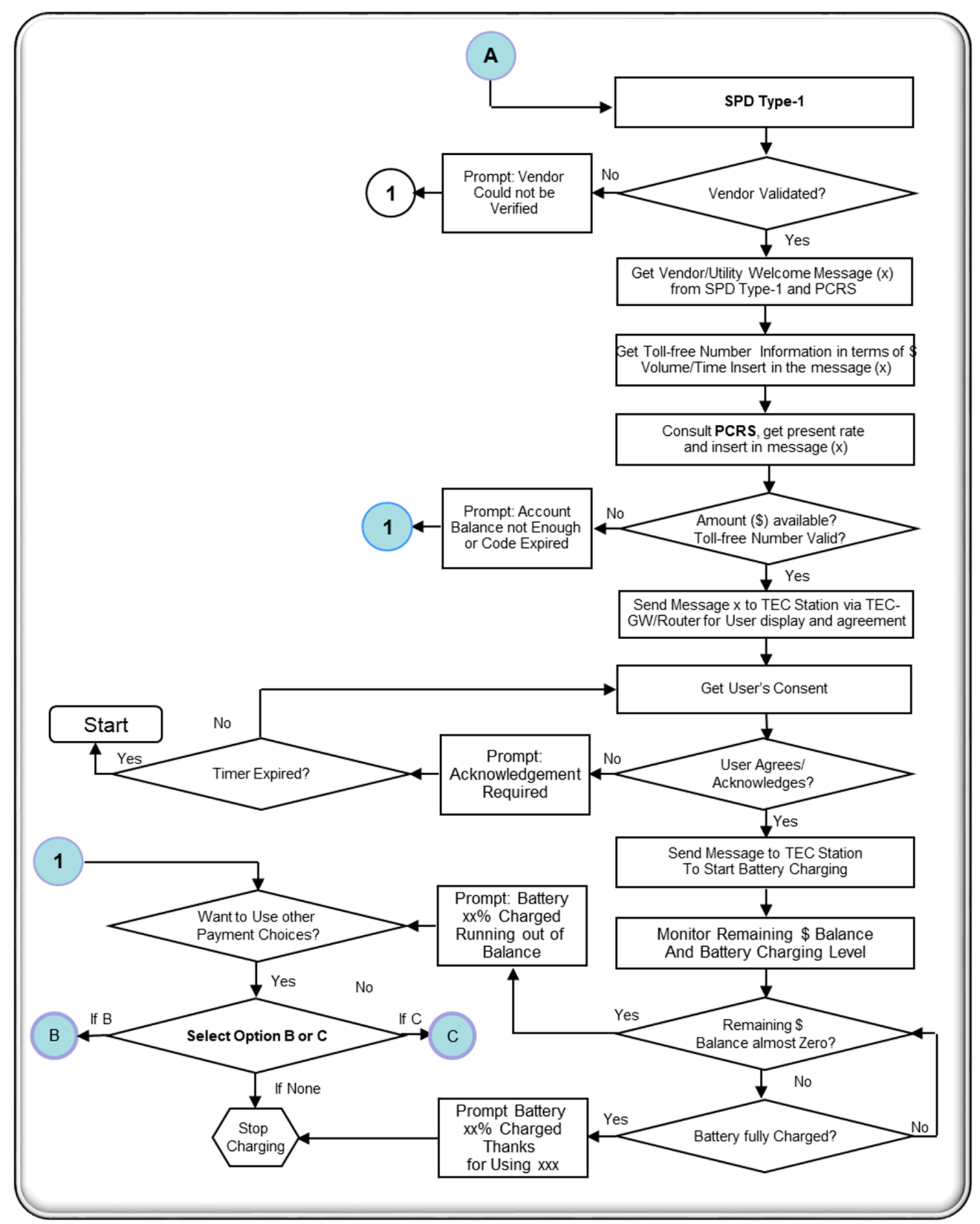

Figure 3. Toll-free service provisioning Type-1 (pre-existing toll-free service offer).

The SPD-Type- 1 contains the database of businesses or communities that participate in offering a toll-free charging service. If the business or community cannot be verified, an error message is sent to the TEC station; otherwise, a customized message for the EV driver is sent for his consent. The message is customized by combining specific service-related information, a memo from the business or community (if any), a memo from the utility (if any), and the service credentials associated with the toll-free number from the SPD Type-1 and the PCRS. 
The algorithm waits for the user's consent for a set period of time. If the user does not provide a response, the algorithm resets and cancels the transaction. On the other hand, upon receiving a positive user response, the toll-free GW/Router sends a message to the TEC station to start battery charging.

When the balance associated with toll-free service reaches an end and the battery is still not fully charged, the TEC station user interface offers three charging choices. The first and second options would be a credit charger to the user's existing account with the utility (where they are charged for domestic usage) or paying through a credit card. This is explained in Figure 4. The third option would be earning credits through watching promotional videos, providing a response to public surveys, etc., as explained in Figure 5.

Figure 4 shows a toll-free service provisioning that is verified by SPD-Type- 2 .

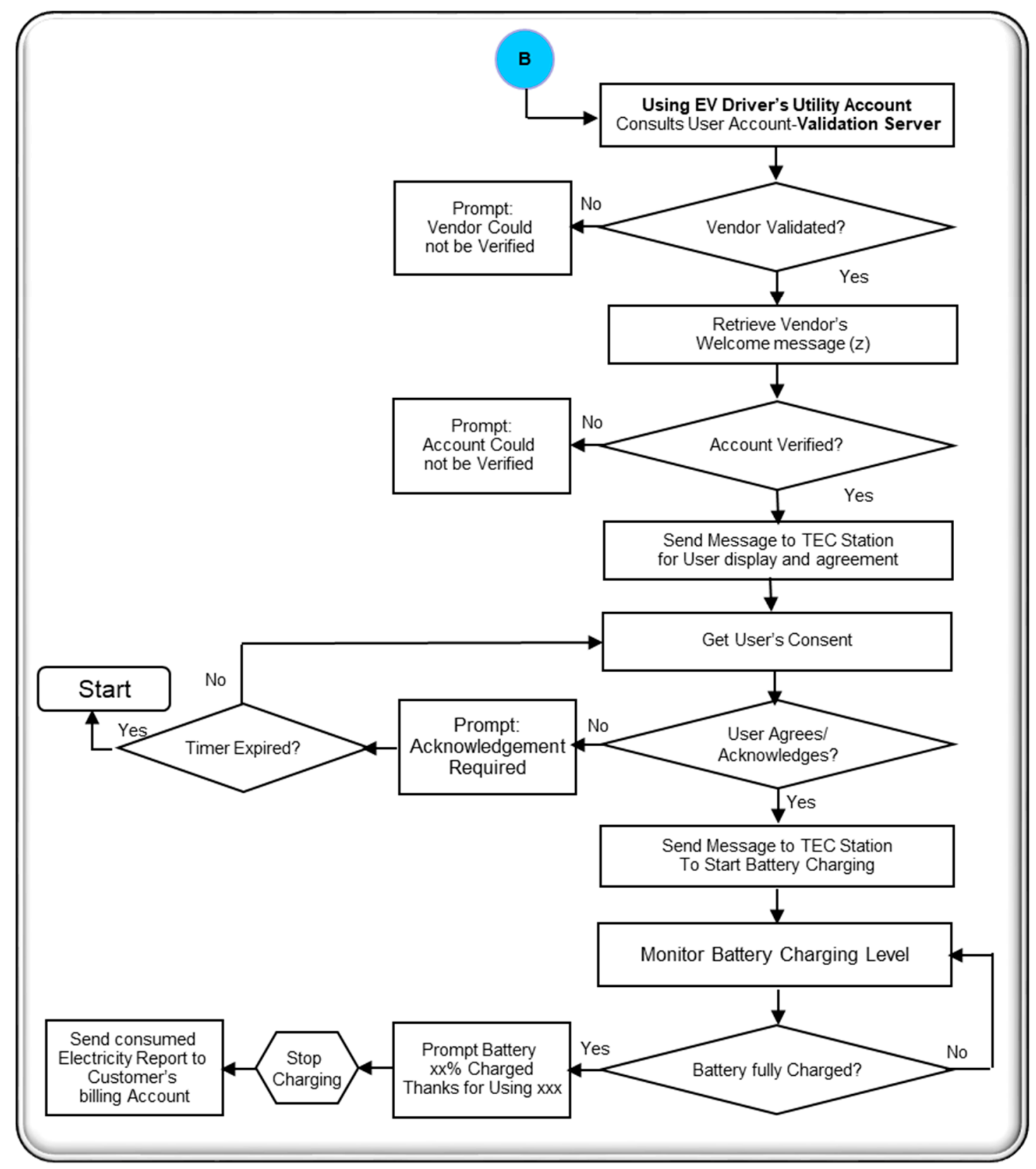

Figure 4. TEC (Toll-Free EV Charging) service provisioning Type-2 (consumer pays). 


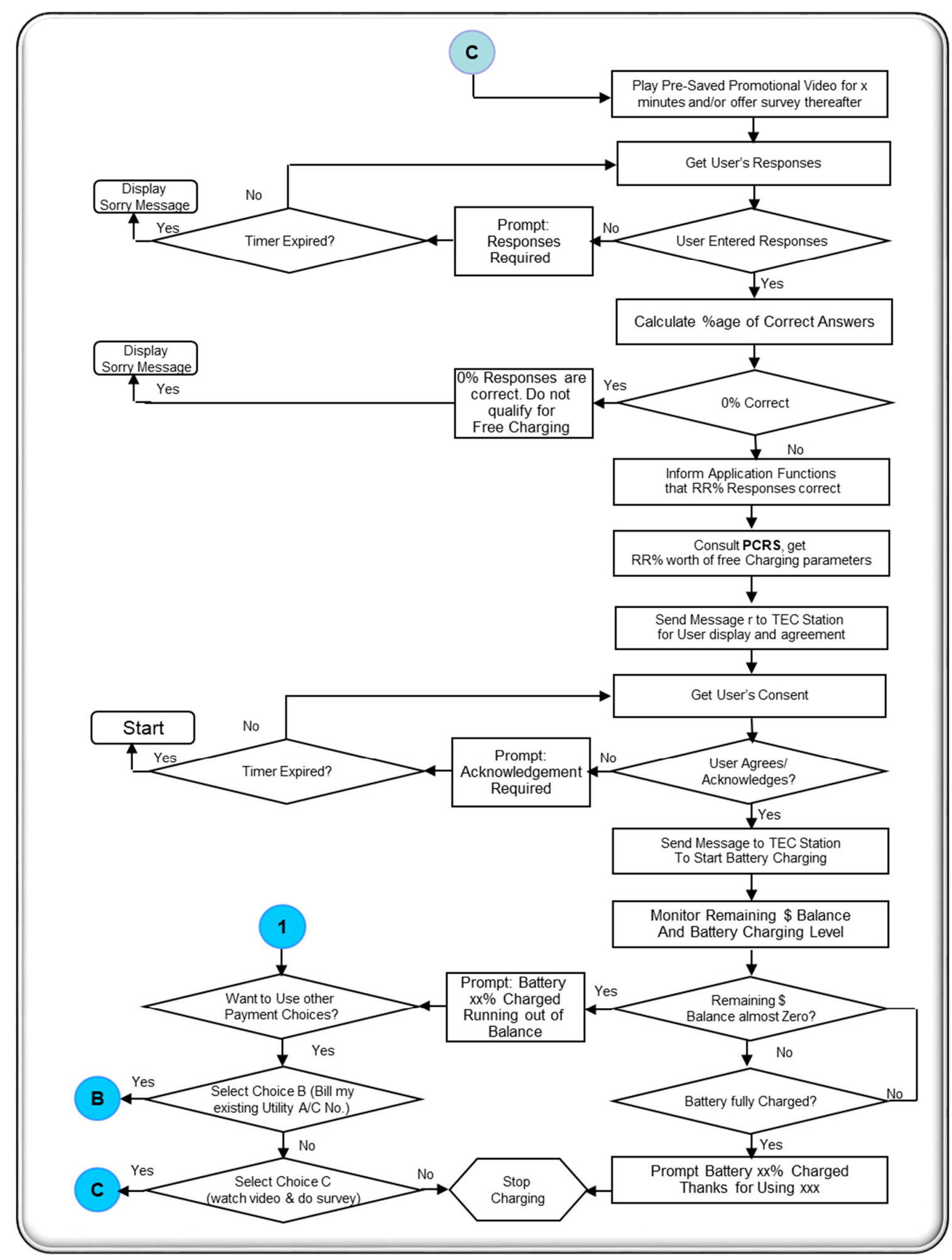

Figure 5. Toll-free service provisioning Type-1 (promotional charging service).

The SPD-Type-2 contains the database of existing consumers' accounts (domestic utility consumers/businesses utility consumers) and offers charging service on a consumer's monthly bill. To do so, the consumer's account is validated. If the consumer's account could not be validated, an error message is sent to the TEC station; otherwise, a message for the EV driver is constructed and 
sent to the user for his consent. This message is constructed by consulting SPD Type- 2 and PCRS and contains the utility memo and service credentials associated with a toll-free number (account number).

Just as with SPD-Type-1 transactions, the algorithm waits for the user's consent for a specified period of time. If the user does not provide a response, the algorithm resets and the current transaction is canceled. Upon receiving a positive response, the toll-free GW/Router sends a message to the TEC station to start battery charging.

The toll-free-GW/Router coordinates the whole process and routes messages related to charging cessation and giving corresponding updates to the user. The billing records are sent to the appropriate billing entities.

Figure 5 is a continuation of Figure 3. It shows toll-free service provisioning by earning credits through watching promotional videos or providing a response to public surveys, etc.

In this scenario, pre-saved promotional video(s) are played for $\mathrm{x}$ minutes and/or thereafter, a survey/questionnaire may be presented (the algorithm offers the survey/promotional video that may be completed in a time equal to the battery charging time). Upon the EV driver's consent, the algorithm may proceed as shown in Figure 4. Based on the user's correct or attempted responses, the equivalent extent of toll-free charging is offered.

The promotion-based service scenario is coordinated by the TEC-GW/Router and the application function.

The TEC-GW/Router receives the service value (in terms of the monetary amount) from the application hosting network and the service cost from the host utility network. It then computes the service corresponding service credentials (charging duration or volume $(\mathrm{kWh})$ ) and offers the toll-free service to the EV Driver through the TEC station. The application function may reside anywhere in the network; however, it must have a business agreement with the utility company. The application function keeps a promotional database (such as promotional videos and surveys) and negotiation parameters (such as the amount of time the promotional video was played, the number of correct responses, equivalent rewards, etc.) and it informs the TEC-GW/Router about the availability of any promotional service options for the end user.

\section{ISO15118 (Existing Standard) and IEC63110 (Future Standard) for Back-Office Communication}

As shown in Figure 1, The EV makes a physical connection with the TEC station (more precisely, to the Electric Vehicle Supply Equipment (EVSE)) using ISO/IEC 15,118 standard over an EV charging cable. The complete message sequence for front-end communication between EV and EVSE for EV charging can be found in [10]. The TEC (more precisely, Supply Equipment Communication Controller (SECC)) communicates with the utility TEC GW/Router to control the overall charging procedure. The complete message sequence for back-office communication between SECC and TEC GW/Router uses ISO/IEC 61,850 and a newly developed standard, IEC63110. The description of the former can be found in [11] and the description of the latter can be found in [12].

To calculate the cost per mile, following mathematical relation, as explained in [13] can be used, i.e., Cost/Mile of EV $=\frac{\text { Cost of Electricty?Consumption of electricity to travel } 100 \text { miles }}{100}$. Thus, for example, the Cost $/$ Mile of $\mathrm{EV}=\$ 0.374$, if electricity costs $\$ 0.11 / \mathrm{kWh}$ and the vehicle consumption is $34 \mathrm{kWh}$ to travel 100 miles.

\section{Future Work}

To make the system capable of automatically billing multiple toll-free numbers/accounts during a single charging event (wherein multiple toll-free numbers/accounts are validated, and the maximum charging duration is predetermined).

To make the system capable of supporting other value-added services (such as car performance diagnostic service, self-diagnostic, self-service, etc.) in collaboration with car dealers or any auto workshops. This is possible because the EV, while charging, is connected via its communication 
channel in addition to its electrical channel. Communication channels can be used to run a remote computer diagnostic test to find glitches in a car's performance.

Acknowledgments: The author is highly grateful to his department head, Kaveh Heidary for his guidance and support, and also time and interest in reviewing the manuscripts, that improved the paper.

Conflicts of Interest: The author declares no conflict of interest.

\section{Abbreviations}

$\begin{array}{ll}\text { AAMU } & \text { Alabama A\&M University } \\ \text { DHCP } & \text { Dynamic Host Configuration Protocol } \\ \text { IPSec } & \text { Internet Protocol Security } \\ \text { IETF } & \text { Internet Engineering Task Force } \\ \text { SSL } & \text { Secure Socket Layer } \\ \text { TEC-GW } & \text { Toll-Free EV Charging-Gateway/ Router } \\ \text { IP } & \text { Internet Protocols } \\ \text { TEC } & \text { Toll-Free EV Charging } \\ \text { TEC-AAA } & \text { Toll-Free EV Charging-Authentication, Authorization and Accounting Server } \\ \text { TVA } & \text { Tennessee Valley Authority } \\ \text { SSH } & \text { Secure Shell } \\ \text { PCRS } & \text { Policy and Charging Rules Server } \\ \text { NFC } & \text { Near Field Communication } \\ \text { TLS } & \text { Transport Layer Security } \\ \text { SPD } & \text { Subscription Profile Database } \\ \text { SCE } & \text { Southern California Edison } \\ \text { EV } & \text { Electric Vehicle } \\ \text { KWh } & \text { KiloWatt Hour } \\ \text { RFID } & \text { Radio Frequency ID } \\ \text { GW } & \text { Gate Way }\end{array}$

\section{References}

1. HIS Markit. Global EV Charging Stations to Skyrocket by 2020, IHS Report Says. Available online: http:/ / news.ihsmarkit.com/press-release/automotive/global-ev-charging-stations-skyrocket-2020ihs-report-says (accessed on 17 February 2019).

2. DigitalPhone.io. Toll-Free Numbers. Available online: https://digitalphone.io/resources/toll-freenumbers / (accessed on 17 February 2019).

3. Lifewire. What is an IP Address. Available online: https://www.lifewire.com/what-is-an-ip-address2625920 (accessed on 17 February 2019).

4. EV Charging Systems Security Requirements for European Networks Cyber Security. Available online: https://www.elaad.nl/uploads/downloads/downloads_download/Security_Requirements_ Charge_Points_v1.01_august2017.pdflist (accessed on 17 February 2019).

5. Mustafa, M.A.; Zhang, N.; Kalogridis, G.; Fan, Z. Smart Electric Vehicle Charging: Security Analysis. In 2013 IEEE PES Innovative Smart Grid Technologies Conference, ISGT; 2013; pp. 1-6. Available online: https:/ / www.sis.se/api/document/preview/917223/ (accessed on 17 February 2019). [CrossRef]

6. Dynamic Host Configuration Protocol, RFC No. 2131. Available online: https://tools.ietf.org/html/rfc2131 (accessed on 17 February 2019).

7. Halim, F.; Yussof, S.; Rusli, M.E. Cyber Security Issues in Smart Meter and Their Solutions. Int. J. Comput. Sci. Netw. Secur. 2018, 18, 99-109.

8. Authentication, Authorization, and Accounting (AAA) Transport Profile, IETF RFC 3539. Available online: https: / tools.ietf.org/html/rfc3539 (accessed on 17 February 2019).

9. DIAMETER Protocol, RFC No. 6733. Available online: https://tools.ietf.org/html/rfc6733 (accessed on 17 February 2019).

10. ISO 15118-2. Available online: https://www.sis.se/api/document/preview/917223/ (accessed on 17 February 2019). 
11. Matousek, P. Description of IEC 61850 Communication. Available online: http:/ / www.fit.vutbr.cz/research/ pubs /index.php?file=\%2Fpub\%2F11832\%2FTR-61850.pdf\&id=11832 (accessed on 17 February 2019).

12. Standard IEC63110-Standardizing the Management of Electric Vehicle (Dis-)Charging Infrastructures. Available online: https:/ / www.iec.ch/dyn/www /f?p=103:30:5986558079731::::FSP_ORG_ID,FSP_LANG_ ID:1255,25 (accessed on 17 February 2019).

13. Alternative Fuels Data Center, by Department of Energy. Available online: https:/ / afdc.energy.gov/fuels / electricity_charging_home.html (accessed on 17 February 2019).

(C) 2019 by the author. Licensee MDPI, Basel, Switzerland. This article is an open access article distributed under the terms and conditions of the Creative Commons Attribution (CC BY) license (http:/ / creativecommons.org/licenses/by/4.0/). 